\title{
A CONVERSE OF THE JORDAN-BROUWER SEPARATION THEOREM IN THREE DIMENSIONS*
}

\author{
BY
}

\author{
R. L. WILDER
}

That the closed ( $n-1)$-manifold immersed in euclidean space, $E_{n}$, of $n$ dimensions $(n>2)$, separates $E_{n}$ into just two domains of which it is the common boundary, was shown by Brouwer in 1912. $\dagger$ That the points of the manifold are accessible from each of its complementary domains Brouwer proved in an accompanying paper, $\ddagger$ and in the latter connection he gave an example to show that a bounded and closed point set which separates $E_{n}$ into just two domains and every point of which is accessible from each of these domains, is not necessarily homeomorphic with a closed manifold.

The above results of Brouwer, in so far as the connectivity of the set residual to the manifold in $E_{n}$ is concerned, received considerable extension at the hands of $\mathrm{J}$. W. Alexander, who not only demonstrated, using modulo 2 Betti numbers, that the residual set is just two connected domains, but that a certain duality exists between the connectivity numbers of the set and those of its complement. $\S$ If we denote the $i$ th connectivity number of a set $F$ by $R^{i}(F)$, then, for the particular case where $C^{i}$ is a set in $E_{n}$ homeomorphic with an $i$-sphere, Alexander showed that

$$
\begin{aligned}
& R^{i}\left(C^{i}\right)=R^{n-i-1}\left(E_{n}-C^{i}\right)=2, \\
& R^{s}\left(C^{i}\right)=R^{n-s-1}\left(E_{n}-C^{i}\right)=1
\end{aligned}
$$

In the case where $n=3$ and $i=2$, relation (1) states that the complement of a simple closed surface in $E_{3}$ is just two domains (as also shown by Brouwer),

* Presented to the Society, December 31, 1928, March 29, 1929, and August 30, 1929; received by the editors October $11,1929$.

$\dagger$ L. E. J. Brouwer, Beweis des Jordanschen Satzes für den n-dimensionalen Raum, Mathematische Annalen, vol. 71 (1912), pp. 314-327.

$\ddagger$ L. E. J. Brouwer, Über Jordansche Mannigfaltigkeiten, ibid., pp. 320-327.

$\S \mathrm{J}$. W. Alexander, $A$ proof and extension of the Jordan-Brouwer separation theorem, these Transactions, vol. 23 (1922), pp. 333-349. Familiarity with this work is assumed throughout the present paper.

The terminology "Betti number" is at present being employed more and more to denote connectivity numbers that are uniformly less by one unit than the numbers $R^{i}$ of the present paper. However, since the present paper is based so much upon the above paper of Alexander, it seems advisable to retain the connectivity numbers as defined in that connection (although they are not termed "Betti numbers" by Alexander). 
and relation (2) states the important additional fact that every closed $s$-chain (where $s<2$ ) in the complement bounds an $(s+1)$-chain in the complement. Thus, although as Alexander pointed out in another connection* the domains complementary to a simple closed surface in $E_{3}$ may not be simply connected (may, in fact, require an infinite number of generators), every closed 1-chain in either of the domains bounds in that domain.

In contemplating so simple a surface as the torus, it becomes apparent that in formulating a converse of the Jordan-Brouwer separation theorem the relation (2) must be taken into account as well as relation (1). On the other hand, a glance at Brouwer's example cited above is sufficient to inform one that even with the duality relations (1) and (2), the accessibility of the points of a surface from each of its complementary domains is not enough to ensure that surface being, in $E_{3}$, a simple closed surface. Some condition must be imposed that will do away with the crinkliness of the surface. It occurred to the author that such a condition might be the following: If $K$ is a simple closed surface in $E_{3}, D$ one of the domains complementary to $K$, and $\epsilon$ a positive number, then there exists a positive number $\delta$ such that if $\Gamma^{0}$ is a closed 0 -chain in $D$ of diameter less than $\delta$, then $\Gamma^{0}$ bounds an open 1-chain $\Gamma^{1}$ in $D$ whose diameter is less than $\epsilon$. This property of $D$ may be concisely expressed by saying that the 0 -chains of $D$ are uniformly homologous to zero in $D$.

It will be shown in $\$ 1$ of this paper (which may be considered as complementary to Alexander's paper on the Jordan-Brouwer separation theorem, since it utilizes the machinery introduced by him in order to treat accessibility properties into which he did not go), that the above condition is a necessary condition for the general $n$-dimensional case, and in $\$ 3$ it will be shown that together with part of Alexander's duality relation it enables one to give, in $E_{3}$, a converse of the Jordan-Brouwer theorem.

The chief difficulty encountered by the author in applying the above, essentially combinatorial, properties, $\uparrow$ to characterizing the sphere immersed in $E_{3}$, was that the known characterizations of the simple closed surface

${ }^{*} \mathrm{~J}$. W. Alexander, An example of a simply connected surface bounding a region which is not simply connected, Proceedings of the National Academy of Sciences, vol, 10 (1924), pp. 8-10.

$\dagger$ It will be shown below, in the Appendix, that uniformly homologous to zero as a condition relative to the 0 -chains of a domain is equivalent to the condition uniform connectedness im kleinen for the domain.

$\ddagger$ The earliest characterization of which the author knows was announced by R. L. Moore and J. R. Kline, their abstract being in the Bulletin of the American Mathematical Society, vol. 28 (1922), p. 380. The same definition is given by Miss I. Gawehn (except for the greater generality of the space considered), in Über unberandete 2-dimensionale Mannigfaltigkeiten, Mathematische Annalen, vol. 98 (1927-28), pp. 321-354. Cf. also C. Kuratowski, Une caractérisation topologique de la surface de la sphire, Fundamenta Mathematicae, vol. 13 (1929), pp. 307-318, as well as abstracts by L. Zippin, Bulletin of the American Mathematical Society, vol. 35 (1929), p. 154 and p. 293. 
were all of a topological type which did not seem to lend themselves very readily to association with the combinatorial properties of the complement. Accordingly a new characterization of the simple closed surface by means of internal properties that are readily associated with the combinatorial properties of the complement was worked out, and is given below in $\$ 2$.

In closing this introduction the author wishes to call to the attention of the reader the problem of giving a converse of the Jordan-Brouwer separation theorem in $E_{3}$ by certain accessibility conditions that are more in the spirit of the Schoenflies converse as formulated in $E_{2},{ }^{*}$ and it is hoped that if the attempt is made to find such conditions, the present work will be of some assistance as having already broken down some of the barriers. For in the opinion of the author the analysis situs relations between closed sets and their complements in spaces of three and higher dimensions will be most easily discovered by first having regard for the connectivity numbers of the complements, $\dagger$ and although it would be unwise to make any predictions, it would certainly seem as though to disregard these in the case of the $(n-1)$-sphere, when already known, in the attempt to obtain equivalent accessibility conditions, would involve an extreme waste of energy.

1. We shall show in this section $\ddagger$ that if $M^{n-1}$ is an $(n-1)$-sphere im-

* In this connection see J. R. Kline, Separation theorems and their relation to recent developments in analysis situs, Bulletin of the American Mathematical Society, vol. 34 (1928), pp. 155-192. See especially pp. 156-159 and top of p. 191. As will be observed later on, regular accessibility is an immediate consequence of the conditions given for the converse in the present paper.

$\dagger$ The recent work of $P$. Alexandroff bears out this opinion strikingly. Cf. his Untersuchungen über Gestalt und Lage abgeschlossener Mengen beliebiger Dimension, Annals of Mathematics, (2), vol. 30 (1928), pp. 101-187. The author wishes to seize this opportunity to express his indebtedness to Dr. Alexandroff, contact with whom, both through his memoirs and personally (during a recent brief visit at the University of Michigan), directed the attention of the author to the possibility of associating continuity and combinatorial methods in treating the problems of analysis situs.

$\ddagger$ The content of this section was presented, along with certain other results, to this Society, March 29, 1929, under separate title (cf. Bulletin of the American Mathematical Society, vol. 35, p. 458, abstract No. 22). It is felt, however, that the theorems and corollaries given above properly belong in the present paper. As for the other results of the paper just referred to, the theorem that the points of an $i$-cell $(0 \leqq i \leqq n-1)$ are regularly accessible from the complement in $E_{n}$ is a special case of a more recent theorem established by the author, to the effect that in $E_{n}$ the points of any closed set which is homeomorphic with a subset of $E_{n-1}$ are regularly accessible from the complement; several of the other results were found to duplicate already known theorems and were withdrawn from the announcement in abstract. One of the things so deleted was an example which the author believes to be not without interest, and which he has not seen in the literature, viz., an arc in $E_{3}$ which cannot lie on, or bound, any set homeomorphic with a 2-cell, and which cannot be thrown into a straight line interval by a (1-1)-continuous transformation of $E_{3}$ into itself. (Cf. L. Antoine, Sur. l'homéomorphie de deux figures et de leurs voisinages, Journal de Mathématiques, (8), vol. 4 (1921), pp. 221-325.) The chief interest of the example is its ease of description: On a great circle of the 2-sphere, let $A B C D E A$ be points in the cyclical order named, and let the portion $B C$ of the 
mersed in $E_{n},{ }^{*}$ and $D$ is either of its complementary domains, then the 0 -chains of $D$ are uniformly homologous to zero in $D$. Consequently the domain $D$ is uniformly connected im kleinen (see Appendix) - a fact which was established for the case where $n=2$ and $M^{n-1}$ is a 1 -sphere by $\mathrm{R} . \mathrm{L}$. Moore. $\dagger$ As a corollary it follows that the points of the sphere are not only accessible, but are regularly accessible, from the complementary domains.

THEOREM 1. Let $M^{n-1}$ be an $(n-1)$-sphere immersed in $E_{n}$, and let $D$ be one of the domains of $E_{n}-M^{n-1}$. Then the 0-chains of $D$ are uniformly homologous to zero in $D$.

Let the domain $E_{n}-\left(M^{n-1}+D\right)$ be denoted by $D_{1}$.

Suppose the 0 -chains in $D$ are not uniformly homologous to zero. Then it easily follows that there is a point $P$ on $M^{n-1}$ and a spherical neighborhood $U$ of $P$, such that in every neighborhood of $P$ there is a 0 -chain which does not bound in that part of $D$ which lies in $U$. We shall denote the frontier of $U$ by $F$.

There is a cell $C^{n-1}$ of $M^{n-1}$ which contains $P$ as a non-boundary point and lies wholly in $U$. Denote the boundary of $C^{n-1}$ by $C^{n-2}$, and let the set of all those points of $M^{n-1}$ that are not interior points of $C^{n-1}$ be denoted by $B^{n-1}$. Let $V$ be a spherical neighborhood of $P$ of such a radius that $V$ contains no points of $B^{n-1}$. Let $x_{1}^{0}$ and $x_{2}^{0}$ be any two 0 -cells of $D$ that lie in $V$. Since the closed chain $x_{1}^{0}+x_{2}^{0}$ bounds a 1-chain $L_{B}{ }^{1}$ interior to $V$, we have

$$
x_{1}^{0}+x_{2}^{0} \sim 0 \quad\left[\bmod 2, E_{n}-\left(F+B^{n-1}\right)\right] .
$$

In order to deal with a specific case, we may assume that a 0 -cell, $y_{1}{ }^{0}$, of $L_{B}{ }^{1}$, lies in $D_{1} ; L_{B}{ }^{1}$ having been obtained, say, by joining $x_{1}{ }^{0}$ and $x_{2}^{0}$ to such a cell by chains $L_{B_{1}^{1}}^{1}$ and $L_{B_{2}}^{1}$, respectively.

arc $A B C D$ be replaced by a new portion containing two infinite sequences of simple trefoil knots (i.e., knots in the sense that the great circle becomes knotted), which of course have to leave the 2 -sphere, and which respectively approach the points $B$ and $C$ as limiting points. This can be done in such a way that the new portion $B C$ is still an arc from $B$ to $C$ that does not meet the $\operatorname{arc} B A E D$ of the great circle. Then the portions $A B$ and $C D$ of the great circle, together with the new portion $B C$, is an arc of the type described above.

* I.e., $M^{n-1}$ is a point set which is in (1-1)-continuous correspondence with an $(n-1)$-sphere, and is therefore not necessarily formed by cells of the subdivisions of $E_{n}$ which are used in the congruences and homologies of the proof. Cf. J. W. Alexander, these Transactions, loc. cit., for definitions of chain, homology, etc. The reader should be on his guard in the matter of terminology which has various meanings depending upon its application in a point-set theoretic sense or in a combinatorial sense. (E.g., "chain," "closed," "open," etc. Present usage seems to apply the term "chain" only to open chains, chains that are closed being termed "cycles." We are retaining the terminology of Alexander's paper, however, in the present connection.)

$\dagger$ R. L. Moore, A characterization of Jordan regions by properties having no reference to their boundaries, Proceedings of the National Academy of Sciences, vol. 4 (1918), pp. 364-370. 
On the other hand, as every 0 -chain in $D$ bounds in $D$, even though it may not bound im kleinen, there is a chain $L_{C}{ }^{1}$ in $D$ which is bounded by the chain $x_{1}{ }^{0}+x_{2}{ }^{0}$. Hence,

$$
x_{1}^{0}+x_{2}^{0} \sim 0 \quad\left(\bmod 2, E_{n}-C^{n-1}\right) .
$$

If now we can show that the closed chain

$$
L_{B}{ }^{1}+L_{C}{ }^{1} \sim 0 \quad\left(\bmod 2, E_{n}-C^{n-2}\right),
$$

it will follow from relations (3), (4) and (5), and Alexander's Corollary $W^{i}$, that $x_{1}{ }^{0}+x_{2}{ }^{0}$ bounds in the set common to $D$ and $U$, and since no restriction is placed upon the choice of $x_{1}{ }^{0}$ and $x_{2}{ }^{0}$, a contradiction of the supposition that the 0 -chains of $D$ are not uniformly homologous to zero will be obtained. We therefore proceed to demonstrate the validity of relation (5).

Let $C_{B^{n-1}}$ be an arbitrary cell of $M^{n-1}$ interior to $B^{n-1}$. Then $x_{1}^{0}$ and $y_{1}^{0}$ bound a 1-chain $K_{1}{ }^{1}$ in $E_{n}-\left(M^{n-1}-C_{B^{n-1}}\right)$. The closed chain $K_{1}{ }^{1}+L_{B 1^{1}}$ links $C^{n-2}$, for if it did not, then, by virtue of Alexander's Corollary $W^{i}$, $x_{1}^{0}+y_{1}{ }^{0}$ would bound in $E_{n}-M^{n-1}$. Similarly, the closed chain $K_{1}{ }^{1}+L_{c^{1}}+$ $L_{B 2}{ }^{1}$ links $C^{n-2}$. However, by relation (1) there is only one linearly independent non-bounding 1-chain in $E_{n}-C^{n-2}$. Hence

$$
K_{1}^{1}+L_{B 1}{ }^{1} \sim K_{1}^{1}+L_{C}{ }^{1}+L_{B 2}{ }^{1} \quad\left(\bmod 2, E-C^{n-2}\right) .
$$

Since homology (6) implies homology (5), the theorem is proved.

Corollary 1. The points of an $(n-1)$-sphere, $M^{n-1}$, immersed in $E_{n}$, are regularly accessible $\dagger$ from the complement of the sphere.

Let $P$ be a point of $M^{n-1}$, and $D$ one of the domains complementary to $M^{n-1}$. Since there are only two domains in the complement of $M^{n-1}$, it will be sufficient to prove $P$ regularly accessible from $D$.

By virtue of Theorem 1 there exists (1) a sequence of spherical neighborhoods of $P, U_{1}, U_{2}, U_{3}, \cdots$, such that if $r_{k}$ is the radius of $U_{k}$, then $r_{k}>r_{k+1}$, and $\lim _{k \rightarrow \infty} r_{k}=0$; and such that for $k>1$, any closed 0 -chain of $D$ that lies in $U_{k}$ bounds a 1-chain that lies wholly in the common part of $U_{k-1}$ and $D$; (2) a sequence of 0 -cells $x_{2}{ }^{0}, x_{3}{ }^{0}, x_{4}{ }^{0}, \cdots$, such that, for every $k, x_{k}{ }^{0}$ lies in the common part of $D$ and $U_{k}$.

* These Transactions, loc. cit., p. 342.

$\dagger$ A point $P$ is said to be regularly accessible from a point set $D$ if for every positive number $\epsilon$ there exists a positive number $\delta$ such that if $Q$ is a point of $D$ whose distance from $P$ is less than $\delta$, then there is an arc $P Q$ in $D+P$ whose diameter is less than $\epsilon$. Cf. G. T. Whyburn, Concerning the open subsets of a plane continuous curve, Proceedings of the National Academy of Sciences, vol. 13 (1927), pp. 650-657. 
For any $k, x_{k}^{0}$ and $x_{k+1}^{0}$ bound a 1-chain, $\Gamma_{k}^{1}$, which lies wholly in the common part of $D$ and $U_{k-1}$. As the set of points

$$
M=\sum_{k=2}^{\infty} \Gamma_{k}^{1}+P
$$

is a continuous curve, there is an arc from $x_{2}{ }^{0}$ to $P$ that lies in $M$, and hence (except for $P$ ) in the common part of $U_{1}$ and $D$. As the first neighborhood, $U_{1}$, is arbitrary, and as in every neighborhood of a point $x$ of $D$ in $U_{2}$ there can be found a 0 -cell $x_{2}{ }^{0}$ in $D$, the corollary is proved.

If, in the proof of Theorem $1, M^{n-1}$ denotes an $(n-1)$-manifold, and $P$ denotes a point which is interior to (i.e., not on the boundary of) a cell $C^{n-1}$ which is one of the cells defining $M^{n-1}$, then the argument to show that $x_{1}{ }^{0}+x_{2}{ }^{0}$ is homologous to zero in $D \cdot U$ goes through as before. However, if $P$ is such a point of the manifold that it cannot lie interior to any such cell, then the above 0 -chain may fail to bound in $D \cdot U$. (Cf., for instance, the set $K$ defined in the Appendix, paragraph beginning "Condition (3)".) Hence we can state the following theorem:

Theorem 1a. Let $M^{n-1}$ be any $(n-1)$-manifold immersed in $E_{n}, D$ a domain complementary to $M^{n-1}$, and $P$ a point of $M^{n-1}$ which can be considered as interior to an (n-1)-cell of definition of $M^{n-1}$. Then, if $C^{n-1}$ is any such cell, and $U$ is any spherical neighborhood of $P$ that encloses only points of $M^{n-1}$ that are interior to $C^{n-1}$, there exists a neighborhood $V$ of $P$ such that any 0 -chain in $D \cdot V$ bounds a 1-chain in $D \cdot U$. If every point of $M^{n-1}$ can be considered as lying interior to an $(n-1)$-cell of definition of $M^{n-1}$, the 0 -chains of $D$ are uniformly homologous to zero in $D .{ }^{*}$

Corollary 2. The points of an $(n-1)$-manifold, $M^{n-1}$, immersed in $E_{n}$, are regularly accessible from the complement of the manifold.

If $P$ is an interior point of a cell $C^{n-1}$ of $M^{n-1}$, the proof is the same as that of the above Corollary 1 . If not, then $P$ is on the boundaries of a certain number (at least four) of $(n-1)$-cells of $M^{n-1}$. Let $V$ be any neighborhood of $P$. Since any $(n-1)$-cell of an $(n-1)$-manifold is homeomorphic with an $(n-2)$-sphere and its interior in $E_{n-1}$, there is a neighborhood $U$ of $P$ such that any point of $M^{n-1}$ which lies in $U$ and is interior to a cell $C^{n-1}$ of $M^{n-1}$

\footnotetext{
* In order to avoid confusion in the mind of the reader, it should perhaps be pointed out here that we are using the definition of manifold as given by Alexander in his paper on the Jordan-Brouwer Separation Theorem. That is, an $i$-manifold is a closed $i$-chain whose $i$ th connectivity number has the value 2. Some authors (cf., for instance, Veblen's Analysis Situs, published by this Society, 1922, p. 88) state the additional condition that every point of the $i$-manifold shall have a neighborhood, relative to the manifold, which is an $i$-cell. The definition as given by Alexander of course yields greater generality to the results obtained.
} 
is joined to $P$ by an arc of $M^{n-1} \cdot V$ that lies, except for $P$, wholly interior to $C^{n-1}$ (by Corollary 1). Let $Q$ be any point of $D$ (one of the domains of $E_{n}-M^{n-1}$ ) that lies in $U$. We shall show that there is an arc from $P$ to $Q$ lying, except for $P$, wholly in $D \cdot V$.

Let $P Q$ be any arc of $U$ from $P$ to $Q$, and let $P_{1}$ be its first point on $M^{n-1}$, in the order from $Q$ to $P$. Since in any neighborhood of a point of $M^{n-1}$ there are points of $M^{n-1}$ not on the boundaries of its $(n-1)$-cells, we may assume that $P_{1}$ is interior to a cell $C^{n-1}$ of $M^{n-1}$ whose boundary, $C^{n-2}$, contains $P$. There is an arc, $t$, of $M^{n-1}$, from $P_{1}$ to $P$ and lying wholly interior to $C^{n-1}$, except for $P$, as well as interior to $V$. Let $P_{2}, P_{3}, P_{4}, \cdots$ be a sequence of points of $t$ having $P$ as a sequential limit point, and let $\epsilon$ denote the distance between $t$ and the frontier of $V$ as ordinarily defined for point sets.

Consider the portion $P_{1} P_{2}$ of $t$. Since all points of $P_{1} P_{2}$ are interior points of $C^{n-1}$, it is easy to show that there lies, in $D \cdot V$, an $\operatorname{arc} Q_{1} Q_{2}$ approximating $P_{1} P_{2}$, where $Q_{1}$ is a point of $Q P_{1}$ in the neighborhood of $P_{1}$, and $Q_{2}$ a point in the neighborhood of $P_{2}$. For if $x$ is any point of $P_{1} P_{2}$, there is a number $\delta$ such that any 0 -chain of $D$ in $S(x, \delta)$ bounds a 1-chain of $D$ in $S\left(x, \frac{1}{2} \epsilon\right)$ (Theorem 1a). A simple chain* of the neighborhoods $S(x, \delta)$ from $P_{1}$ to $P_{2}$, together with the joining of 0 -cells in the successive links of the simple chain, yields the arc $Q_{1} Q_{2}$ desired.

By successive approximations to the $\operatorname{arcs} P_{i} P_{i+1}$ by $\operatorname{arcs} Q_{i} Q_{i+1}$ in $D \cdot V$ in such a manner that if $x_{i}$ is any point of $Q_{i} Q_{i+1}, \lim _{i \rightarrow \infty} x_{i}=P$, it is clear that the set $Q Q_{1}+\sum_{i=1}^{\infty} Q_{i} Q_{i+1}+P$ is a continuous curve containing an arc from $Q$ to $P$ and lying, except for $P$, wholly in $D \cdot V$.

\section{2. $\dagger$ We shall now prove the following theorem:}

TheOREM 2. In a metric separable space let $M$ be a compact set containing at least one simple closed curve, and satisfying the following conditions: (1) if $t$ is an arc of $M$, then $M-t$ is connected; (2) if $J$ is a simple closed curve of $M$, then $M-J$ is the sum of two uniformly connected im kleinen $\ddagger$ components. Then $M$ is a simple closed surface; i.e., a set homeomorphic with the sphere $x^{2}+y^{2}+z^{2}=1 . \S$

* Cf. R. L. Moore, On the foundations of plane analysis situs, these Transactions, vol. 17 (1916), pp. 131-164. In particular, see bottom of p. 134, and Theorem 10 of this paper.

$\dagger$ The content of this section was presented to the Society December 31, 1928.

$\ddagger$ A set of points $K$ is said to be uniformly connected im kleinen if for every $\epsilon>0$ there exists a $\delta>0$, such that if $P$ and $Q$ are points of $K$ whose distance apart is less than $\delta$, then $P$ and $Q$ are in a connected subset of $K$ whose diameter is less than $\epsilon$. However, for an open subset of a continuous curve, it is easy to prove that the words "a connected subset" in this definition may be replaced by the words "an arc."

$\S$ When this paper was in process of completion, Dr. Leo Zippin communicated to the author that he had succeeded in showing that a continuous curve $C$ which has the properties that (1) if 
For ease of reference, we shall divide the proof into sections:

I. The set $M$ is connected im kleinen. For if $J$ is a simple closed curve of $M$, and $M_{1}$ and $M_{2}$ are the components of $M-J$, then $M_{1}+J$ and $M_{2}+J$ are connected im kleinen by a theorem of R. L. Moore.* Accordingly the set $M$ is itself connected im kleinen.

II. If $J$ is a simple closed curve of $M$, and $M_{1}$ and $M_{2}$ are the components of $M-J$, then $J$ is identical with the boundary of each of the sets $M_{1}, M_{2}$. For suppose $J$ contains a point, $P$, which is not a limit point of $M_{1}$, say. Then an arc, $t$, of $J$, contains all the boundary points (if any) of $M_{1}$. Then

$$
M-t=M_{1}+\left(M_{2}+J-t\right) .
$$

Since $M_{1}$ and $M_{2}+J-t$ are mutually separated sets, a violation of condition (1) results.

III. The set $M$ is connected. For $M$ contains at least one simple closed curve, $J$, and, by II, $J$ is the common boundary of the components of $M-J$.

IV. If $P$ is a point of $M$, then there is an arc of $M$ which contains $P$, and of which $P$ is not an end point. $\dagger$

Since $M$ is a continuous curve, being a connected im kleinen, closed and connected set, there is an $\operatorname{arc} P P^{\prime}$ of $M$, joining $P$ to some point $P^{\prime}$ of $M-P$. By condition (1) the set $M-P P^{\prime}=D$ is connected. If $P$ is not a limit point of $D$, then there is a point $Q$ of $P P^{\prime}$ distinct from both $P$ and $P^{\prime}$, such that the portion $P Q$ of $P P^{\prime}$ contains no limit points of $D$. Then the set

$$
M-P^{\prime} Q=D+(P Q-Q)
$$

is not connected, since $D$ and $P Q-Q$ are mutually separated sets. But this constitutes a violation of condition (1). Consequently $D$ has $P$ as a limit point.

The set $D+P$ is connected im kleinen. If not, then $P$ is the only point at which it is not connected im kleinen, since $D$ is an open subset of $M$. Then there exists, by Theorem 1 of my paper Characterizations of continuous curves that are perfectly continuous, $\ddagger$ a positive number $\epsilon$ such that for every positive

$t$ is an arc of $C$, then $C-t$ is connected, (2) if $J$ is a simple closed curve of $C$, then $C-J$ is not connected, is a simple closed surface. This definition is an obvious improvement over that given above, and by its use the last two parts of the proof of the theorem of $\$ 3$ can be omitted.

* R. L. Moore, Concerning connectedness im kleinen and a related property, Fundamenta Mathematicae, vol. 3 (1922), pp. 232-237, Theorem 1. The proof given by Moore evidently holds for a compact set in any topological space.

$\dagger$ Note added in proof: Since this paper was submitted to these Transactions, a paper by W. L. Ayres has appeared in the American Journal of Mathematics for October, 1929, in which it is shown (Theorem 10) that if a continuous curve in $E_{n}$ contains no cut point, then the curve is cyclicly connected. As our curve $M$ clearly contains no cut point by virtue of condition (1), it is obvious that the results IV and V follow from Ayres' theorem.

$\ddagger$ Proceedings of the National Academy of Sciences, vol. 15 (1929), pp. 614-621. 
number $\delta<\epsilon$ there is a point $Q$ of $D$ whose distance from $P$ is less than $\delta$ and which is not in the same quasi-component with $P$ of the set of points $(D+P) \cdot S(P, \epsilon) .^{*}$ It follows at once that there is a sequence of points $x_{1}, x_{2}, x_{3}, \cdots$, such that $\epsilon / 2>\rho\left(x_{n}, P\right)>\epsilon / 3$, and such that no two points of this sequence lie in the same quasi-component of $(D+P) \cdot S(P, \epsilon)$. As $M$ is compact, there is a limit point, $x$, of the set of points $\sum_{n=1}^{\infty} x_{n}$. The point $x$ cannot lie in $D$, since $D$ is connected im kleinen. Hence $x$ is on the arc $P P^{\prime}$, and since there is no loss of generality in assuming that $\rho\left(P, P^{\prime}\right)>\epsilon$, we can say that $x$ is distinct from both $P$ and $P^{\prime}$.

However, $D$ is uniformly connected im kleinen in the neighborhood of $x$. For let $t$ be an arc in $P P^{\prime}$ whose end points are distinct from $P$ and $P^{\prime}$, and which contains $x$. Since $M-t$ is connected, by condition (1), there exists in $M-t$, by a theorem of R. L. Moore, $\dagger$ an $\operatorname{arc}$ from $P$ to $P^{\prime}$, and this arc together with the arc $P P^{\prime}$ contains a simple closed curve $J$ which contains $t$, consisting of two arcs, $A x B$ of $P P^{\prime}$ and $A y B$ of $D+A+B$. Let the components of $M-J$ be $M_{1}$ and $M_{2}$, and let $\eta$ be a positive number such that $S(x, \eta)$ contains no points of $J+P P^{\prime}$ that are not points of $t$. Then by condition (2) there is a positive number $\rho$ such that every two points of $M_{1}$ that lie in $S(x, \rho)$ are joined by an arc of $M_{1}$ in $S(x, \eta)$, and a similar statement holds for $M_{2}$. Since all points of $M-J$ in $S(P, \eta)$ belong to $D$, it is clear that if $\eta$ is taken so small that $S(x, \eta)$ is a subset of $S(P, \epsilon)$, the points $x_{1}, x_{2}$, $x_{3}, \cdots$ cannot all lie in distinct quasi-components of $(D+P) \cdot S(P, \epsilon)$.

Accordingly $D+P$ must be connected im kleinen, and by a theorem of Whyburn $\ddagger P$ is regularly accessible from $D$. Hence there is an arc of $M$ containing $P$ and of which $P$ is not an end point.

V. If $P$ is a point of $M$, then $P$ is on some simple closed curve of $M$. This follows at once from IV, and the method used in IV to obtain the simple closed curve $J$.

VI. If $P$ is a point of $M, M-P$ is connected. By V,P is on a simple closed curve $J$ of $M$, and by condition (2) $M-J$ is the sum of two components which have, by II, $J$ as common boundary. Since $M-P$ is the sum of these

* Hereafter, if $P$ is a point and $\epsilon$ any positive number, the symbol $S(P, \epsilon)$ will denote a spherical neighborhood of $P$ of radius $\epsilon$; i.e., the set of all points of the space whose distance from $P$ is less than $\epsilon$. In accordance with the usual notation, $M \cdot N$ denotes the set of points common to $M$ and $N$. By $\rho(P, Q)$ we denote the distance between two points $P$ and $Q$.

$\dagger$ R. L. Moore, Concerning continuous curves in the plane, Mathematische Zeitschrift, vol. 15 (1922), pp. 254-260, Theorem 1. The theorem is evidently true for the case of a continuous curve in the general space we are considering.

$\ddagger \mathrm{G}$. T. Whyburn, Concerning accessibility in the plane and regular accessibility in $n$ dimensions, Bulletin of the American Mathematical Society, vol. 34 (1928), pp. 504-510, Theorem 3. Although stated for $E_{n}$, the proof given for this theorem clearly holds in any metric space. 
components together with some of their common boundary points, it is clear that $M-P$ is connected.

VII. If $M_{1}, M_{2}$ and $J$ are defined as in II, and $A x B$ is an arc such that $<A x B\rangle^{*}$ is in $M_{1}$ and $A+B$ on $J$, then $M_{1}-<A x B>$ is the sum of two components $R_{1}$ and $R_{2}$ whose boundaries are, respectively, $A x B+$ an arc $A y B$ of $J$, and $A x B+$ an arc $A z B$ of $J$, where $A y B$ and $A z B$ have only $A$ and $B$ in common.

Let the two arcs into which $A$ and $B$ divide $J$ be $A y B$ and $A z B$. Let $J_{1}$ and $J_{\mathbf{2}}$ be simple closed curves defined as follows:

$$
\begin{aligned}
& J_{1}=A x B+A y B, \\
& J_{2}=A x B+A z B .
\end{aligned}
$$

By condition (2), $M-J_{1}$ is the sum of two components $R_{1}$ and $R_{1}^{\prime}$ whose common boundary, by II, is $J_{1}$. Let $R_{1}$ be that one of these components which lies in $M_{1}$. (It is clear that $M_{2}$ lies in one of the components of $M-J_{1}$, say in $R_{1}^{\prime}$, and that $R_{1}^{\prime}$ must therefore contain $\langle A z B\rangle$, thus requiring that $R_{1}$ be a subset of $M_{1}$.) Let that one of the components of $M-J_{2}$ that lies in $M_{1}$ be denoted by $R_{2}$.

Suppose that $M_{1}$ contains a point $P$ that is not in the set $R_{1}+R_{2}$ $+\langle A x B\rangle$. Then $P$ lies in $R_{1}^{\prime}$, and if $P_{1}$ is a point of $M_{2}$, there is an arc $P P_{1}$, from $P$ to $P_{1}$, in $R_{1}^{\prime}$. If $Q$ is the first point of $J$ on this arc, in the order from $P$ to $P_{1}$, then $P Q-Q$ lies in $M_{1}$. It is clear that $Q$ is in $\langle A z B\rangle$; let $S(Q, \epsilon)$ be a neighborhood of $Q$ that does not enclose any point of $A x B$. There is a neighborhood $S(Q, \delta)$ such that any two points of $M_{1} \cdot S(Q, \delta)$ are joined in $S(Q, \epsilon)$ by an arc of $M_{1}$. Since points of $P Q-Q$ and $R_{2}$ both lie in $S(Q, \delta)$, and such points are then joined by connected subsets of $M-J_{2}$, a contradiction results from the supposition that $M_{1}$ contains points not in the set $R_{1}+R_{2}+\langle A x B\rangle$. Hence $M_{1}=R_{1}+R_{2}+\langle A x B\rangle$.

VIII. If $P$ is a point of $M$, and $\epsilon$ is any positive number, there is a simple closed curve $J$ in $M$ which does not contain $P$ and such that both $J$ and the component of $M-J$ which contains $P$ lie in $S(P, \epsilon)$.

By V, $P$ is on some simple closed curve $J$ of $M$. Denote the components of $M-J$ by $M_{1}$ and $M_{2}$. We can, of course, suppose that $J$ is not wholly in $S(P, \epsilon)$, and that both $M_{1}$ and $M_{2}$ have points in the exterior of $S(P, \epsilon)$.

By virtue of the uniform connectedness im kleinen of $M_{1}$ and $M_{2}$, together with II, it is easy to show the existence, in $S(P, \epsilon)$, of $\operatorname{arcs} A_{1} x B_{1}$ and $A_{2} y B_{2}$, where $\left\langle A_{1} x B_{1}\right\rangle$ and $\left\langle A_{2} y B_{2}\right\rangle$ lie in $M_{1}$ and $M_{2}$, respectively, and the points $A_{1}, A_{2}, B_{1}, B_{2}$ lie on $J$ in the order $A_{1} A_{2} P B_{2} B_{1} A_{1}$, and such that the arc $A_{1} P B_{1}$ of $J$ lies in $S(P, \epsilon)$. Let the simple closed curve $A_{1} x B_{1}+B_{1} B_{2}+A_{2} y B_{2}$

* By $\langle A x B\rangle$ we denote the set $A x B-A-B$. 
$+A_{1} A_{2}$ (where $A_{1} A_{2}$ and $B_{1} B_{2}$ are portions of $A_{1} P B_{1}$ on $J$ ) be denoted by $J_{1}$. Let that component of $M-J_{1}$ that contains $P$ be denoted by $R_{1}$.

By VII, $\left.M_{1}-<A_{1} x B_{1}\right\rangle$ is the sum of two components, one of which is bounded by the simple closed curve $T_{1}=A_{1} P B_{1}+A_{1} x B_{1}$; denote this component by $K_{1}$. Similarly, that component of $M_{2}$ bounded by the simple closed curve $T_{2}=A_{2} P B_{2}+A_{2} y B_{2}$ denote by $K_{2}$. Then

$$
R_{1}=K_{1}+K_{2}+<A_{2} P B_{2}>\text {. }
$$

For by VII, $R_{1}-<A_{2} P B_{2}>$ is the sum of two components $H_{1}$ and $H_{2}$, boundẹd, respectively, by $T_{1}$ and $T_{2}$. Now $T_{1}$ bounds only two components, one of which contains $M_{2}$. If $H_{1}$ were the latter component, then $H_{1}$ would contain $\left\langle A_{2} y B_{2}\right\rangle$, which is clearly impossible. Then $H_{1}$ is that component of $M-T_{1}$ that does not contain $M_{2}$, i.e., $K_{1}$. Similarly, $H_{2}=K_{2}$. We note that if $\alpha$ is a point of $M_{1}$ that is not in $K_{1}+T_{1}$, then $R_{1}$ does not contain $\alpha$.

If $R_{1}$ does not lie wholly in $S(P, \epsilon)$, there is a point, $P_{1}$, in $R_{1}$, such that $\rho\left(P, P_{1}\right)=\epsilon$.

In a similar manner, we can show the existence, in $S(P, \epsilon / 2)$, of arcs $C_{1} x_{1} D_{1}$ and $C_{2} y_{2} D_{2}$, where $\left\langle C_{1} x_{1} D_{1}\right\rangle$ and $\left\langle C_{2} y_{2} D_{2}\right\rangle$ lie in $K_{1}$ and $K_{2}$, re:spectively, and the end points of these arcs lie on $A_{2} P B_{2}$ in the order $A_{2} C_{1} C_{2} P D_{2} D_{1} B_{2}$. Denote the simple closed curve formed by these two arcs, together with the portions $C_{1} C_{2}$ and $D_{1} D_{2}$ of $A_{2} P B_{2}$, by $J_{2}$. Denote the component of $M-J_{2}$ that contains $P$ by $R_{2}$.

By VII, $\left.K_{1}-<C_{1} x_{1} D_{1}\right\rangle$ is the sum of two components, one of which, $N_{1}$, is bounded by the simple closed curve $F_{1}=C_{1} x_{1} D_{1}+C_{1} P D_{1}$; similarly, that component of $K_{2}-<C_{2} y_{2} D_{2}>$ bounded by the curve $F_{2}=C_{2} y_{2} D_{2}+C_{2} P D_{2}$ denote by $N_{2}$. Then we can show, as above, that

$$
R_{2}=N_{1}+N_{2}+<C_{2} P D_{2}>\text {. }
$$

Consequently, $R_{2}$ is a subset of $R_{1}$, and does not contain the point $\alpha$ defined above.

We may continue in this way indefinitely, obtaining simple closed curves $J_{3}, J_{4}, J_{5}, \cdots$, with corresponding components $R_{3}, R_{4}, R_{5}, \cdots$, all containing $P$, but not $\alpha$, and each component a subset of the preceding; also, requiring that for each positive integer $n$, the curve $J_{n}$ lie in $S(P, \epsilon / n)$.

For some $n, R_{n}$ is a subset of $S(P, \epsilon)$. For if not, there is for every $n$ a point $P_{n}$ of $R_{n}$ such that $\rho\left(P, P_{n}\right)=\epsilon$. Since $M$ is compact, the set of points $\sum_{n=1}^{\infty} P_{n}$ has at least one limit point $Q$, which is in every set $R_{n}$ and such that $\rho(P, Q)=\epsilon$. Common to all the sets $R_{1}, R_{2}, R_{3}, \cdots$, there is a continuum $C$, which contains $P$ and $Q$.

By VI, $M-P$ is connected, and therefore contains an arc $t$ with end 
points $Q$ and $\alpha .^{*}$ There exists a positive integer $k$ such that $\epsilon / k$ is less than the distance between $P$ and the $\operatorname{arc} t$. Then the set $C+t$ is a connected subset of $M-J_{k}$, since $C$ lies in $R_{k}$ and $t$ cannot meet $J_{k}$. However, $P$ lies in $R_{k}$ and $\alpha$ lies in $M-\left(J_{k}+R_{k}\right)$. Thus the supposition that for no $n$ does $R_{n}$ lie in $S(P, \epsilon)$ leads to a contradiction.

The conclusion of Theorem 2 now follows in either one of the following ways: (1) by virtue of the Moore-Kline-Gawehn $\dagger$ definition of the simple closed surface, whose conditions are now seen to be fulfilled; or (2) by virtue of R. L. Moore's Axioms $\Sigma_{1}$ for plane analysis situs. $\ddagger$ For it is easy to see, in view of what has been shown above, that if $Q$ is an arbitrary point of $M$, then $M-Q$ is a topological plane, in that it satisfies the axioms $\Sigma_{1}$. Thus, if $J$ is a simple closed curve of $M-Q$, let that component of $M-J$ which does not contain $Q$ be called a region.

$3 . \S$ We shall prove the following theorem:

Theorem 3 (Converse of the Jordan-Brouwer Separation Theorem in $\left.E_{3}\right)$. Let $K$ be a closed and bounded set in $E_{3}$, such that $E_{3}-K=S_{1}+S_{2}$, where $S_{1}$ and $S_{2}$ are mutually exclusive and

(1) every arc from a point of $S_{1}$ to a point of $S_{2}$ contains at least one point of $K$;

(2) if $P$ is a point of $K$ and $Q$ a point not in $K$, then in every neighborhood of $P$ there is a point $P^{\prime}$ such that there is an arc from $P^{\prime}$ to $Q$ lying except for possibly $P^{\prime}$ wholly in $E_{3}-K$;

(3) the 0 -chains of $S_{i}(i=1,2)$ are uniformly homologous to zero in $S_{i}$; and

(4) the Betti number $(\bmod 2) R^{1}\left(E_{3}-K\right)=1$.

Then $K$ is a simple closed surface.

We shall show that the set $K$ satisfies all the conditions of Theorem 2.

I. The set $K$ is connected. For if not, it is the sum of two mutually separated sets, $K_{1}$ and $K_{2}$. Let $P_{i}$ be a point of $K_{i}(i=1,2)$. By a theorem of Knaster and Kuratowski, $\mathbb{f}$ there exists a continuum $C$ in $E_{3}-K$ which separates $P_{1}$ and $P_{2}$. The continuum $C$ lies wholly in one of the sets $S_{1}, S_{2}$.

* Cf. R. L. Moore, Mathematische Zeitschrift, loc. cit.

† Cf. Bulletin abstract of Moore and Kline, loc. cit., and I. Gawehn, loc. cit.

$\ddagger$ R. L. Moore, On the foundations of plane analysis situs, these Transactions, vol. 17 (1916); pp. 131-164. See also R. L. Wilder, Concerning R. L. Moore's axioms $\Sigma_{1}$ for plane analysis situs, Bulletin of the American Mathematical Society, vol. 34 (1928), pp. 752-760.

$\S$ The contents of this section were presented to the Society August 30, 1929.

I B. Knaster and C. Kuratowski, Sur les ensembles connexes, Fundamenta Mathematicae, vol. 2 (1921), pp. 206-255. The theorem referred to here is Theorem 37, p. 233. This theorem holds true in $E_{n}$, since the theorem of Brouwer used in its proof holds in $E_{n}$, for both bounded and unbounded sets. 
For if it contains points of both $S_{1}$ and $S_{2}$, then $S_{1}$, say, contains a limit point of $S_{2}$. But then, since $K$ is closed, this limit point would be joined to a point of $S_{2}$ by an arc that does not meet $K$, a violation of condition (1). Then $C$ lies in $S_{1}$, say.

Now in the neighborhood of any point $P$ of $K$ there lie points of both $S_{1}$ and $S_{2}$. For if we let $U$ be any neighborhood of $P$ and $x$ a point of $S_{1}$, say, there is, by condition (2), a point $P^{\prime}$ in $U$ which is joined to $x$ by an arc $P^{\prime} x$ and such that $P^{\prime} x-P^{\prime}$ lies in $E_{3}-K$. It is clear that $P^{\prime} x-P^{\prime}$ must lie in $S_{1}$, since $x$ is in $S_{1}$; and as $U$ must contain points of $P^{\prime} x-P^{\prime}$, there is a point of $S_{1}$ in $U$.

Accordingly, in the same domain complementary to $C$ that contains $P_{1}$ there is a point $x_{1}$ of $S_{2}$. By condition (2) there is, in the same domain complementary to $C$ as $P_{2}$, a point $x_{2}$ such that there is an arc $x_{1} x_{2}$ which lies, except possibly for $x_{2}$, wholly in $E_{3}-K$. Then $x_{1} x_{2}-x_{2}$ lies in $S_{2}$, and there is no point of $C$ on $x_{1} x_{2}$, since $C$ is in $S_{1}$. But $x_{1}$ and $x_{2}$ must be separated by $C$ in $E_{3}$. Thus the supposition that $K$ is not connected leads to a contradiction.

II. The set $K$ is connected im kleinen. For if not, it follows from a theorem of R. L. Moore* that there exist two concentric spheres, $R_{1}$ and $R_{2}$, and a sequence of subcontinua of $K$, namely $M_{\infty}, M_{1}, M_{2}, M_{3}, \cdots$, such that (1) each of these continua contains at least one point of $R_{1}$ and $R_{2}$, respectively, but no point exterior to $R_{1}$ or interior to $R_{2}$, (2) no two of these subcontinua have a point in common, and no two of them contain points of any connected subset of $K$ that lies wholly in $R_{1}+R_{2}+I$, where $I$ is the annular domain bounded by $R_{1}$ and $R_{2}$, (3) $M_{\infty}$ is the sequential limiting set of the sequence of continua $M_{1}, M_{2}, M_{3}, \cdots$.

Let $P$ be a point of $M_{\infty}$ in $I$, and let $R$ be a spherical neighborhood of $P$ such that $R^{\prime}$ lies in $I$ (where $R^{\prime}$ denotes $R$ together with its boundary). Let us consider $R$ as a space, $\bar{E}_{3}$, and if $M$ is a point set, let us denote the product $M \cdot R$ by $\bar{M}$.

There exists a positive integer $m$ such that $\bar{M}_{i}$, for $i \geqq m$, is non-vacuous. The set $\bar{K}$ is closed relative to $\bar{E}_{3}$, and by condition (2) of Moore's theorem just quoted, no connected subset of $\bar{K}$ joins $\bar{M}_{i}$ and $\bar{M}_{j}(i \neq j ; i, j \geqq m)$. There exists a spherical neighborhood, $U$, of $P$ in $\bar{E}_{3}$, such that any 0 -chain of $S_{i}$ in $U$ is homologous to zero in $\bar{S}_{i}$. Let $i$ and $j$ be values such that $\bar{M}_{i}$

* R. L. Moore, A characterization of Jordan regions by properties having no reference to their boundaries, Proceedings of the National Academy of Sciences, vol. 4 (1918), pp. 364-370. The theorem referred to here is not given any explicit statement, in theorem form, in this paper, but will be found in such form in the same author's Report on continuous curves from the viewpoint of analysis situs, Bulletin of the American Mathematical Society, vol. 29 (1923), pp. 296-297. 
and $\bar{M}_{i}$ have points $P_{i}$ and $P_{j}$, respectively, in $U$. There exists a continuum, $C$ (continuum relative to $\bar{E}_{3}$ ) that lies in $\bar{E}_{3}-\bar{K}$ and separates $P_{i}$ and $P_{j}$ in $\bar{E}_{3}$. For there is a separation of $\bar{K}$ into two mutually separated sets containing $\bar{M}_{i}$ and $\bar{M}_{j}$, respectively,* and the theorem of Knaster and Kuratowski used in I applies in $\bar{E}_{3}$.

Now, as shown in a similar case in I, $C$ must lie wholly in, say, $S_{1}$. But in every neighborhood of $P_{i}$, and of $P_{j}$, there are points of $S_{2}$; in particular, there are such points in neighborhoods of $P_{i}$ and $P_{j}$ that lie in $U-C \cdot U$, and these bound 1-chains of $\bar{S}_{2}$. As such chains must contain points of $C$, and hence of $S_{1}$, a contradiction is established, and $K$ must be connected im kleinen.

III. The sets $S_{1}$ and $S_{2}$ are connected. Consider $S_{1}$, and let $x$ and $y$ be any two of its points. Let $P$ be any point of $K$, and let $U_{1}$ be a spherical neighborhood of $P$. By condition (3) there is a spherical neighborhood $U_{2}$ of $P$ such that if $a^{0}$ and $b^{0}$ are two 0 -cells of $S_{1}$ in $U_{2}$, then $a^{0}$ and $b^{0}$ bound a 1 -chain of $S_{1}$ in $U_{1}$. Now, as already shown, $U_{2}$ contains two points, $x_{1}$ and $y_{1}$, which are joined to $x$ and $y$, respectively, by $\operatorname{arcs} x x_{1}$ and $y y_{1}$ in $S_{1}$. There is no loss of generality in assuming that $x_{1}$ and $y_{1}$ are 0 -cells of the subdivision of $E_{3}$, and accordingly bound a 1-chain $L^{1}$ of $S_{1}$ in $U_{1}$. Then $x x_{1}+L^{1}+y y_{1}$ is a connected subset of $S_{1}$ joining $x$ and $y$, and therefore $S_{1}$ is connected.

IV. No arc of $K$ disconnects $K$. For let $t$ be an $\operatorname{arc}$ of $K$ and suppose that $K-t=K_{1}+K_{2}$, where $K_{1}$ and $K_{2}$ are mutually separated sets. The sets $K_{1}+t$ and $K_{2}+t$ are closed.

Let $P$ and $Q$ be points of $S_{1}$ and $S_{2}$, respectively, which are also 0 -cells of the subdivision of $E_{3}$. By condition (2), $K_{1}+t$ does not separate $P$ and $Q$ in $E_{3}$; let $L_{1}{ }^{1}$ be a 1 -chain bounded by $P$ and $Q$ in the complement of $K_{1}+t$. Similarly, let $L_{2}{ }^{1}$ be a 1 -chain bounded by $P$ and $Q$ in the complement of $K_{2}+t$. Since the closed 1-chain $L_{1}^{1}+L_{2}^{1}$ cannot link the arc $t$, $\dagger$ it follows from a theorem of $\mathrm{J}$. W. Alexander $\dagger$ that $P+Q$ bounds a 1 -chain in $E_{3}-K$; in other words, condition (1) is violated. Hence $t$ does not separate $K$.

$V$. If $J$ is a simple closed curve of $K$, then $K-J$ is not connected. Suppose that $K-J$ is connected.

By the Alexander duality relation (see introduction) there is a closed 1-chain $\Gamma^{1}$ which links $J$. We shall first show that $\Gamma^{1}$ has points in both $S_{1}$ and $S_{2}$.

Suppose $\Gamma^{1}$ is contained in $S_{1}+K$. Then it is clear that $\Gamma^{1}$ contains points of $K$, for if not, by condition (4) we should have

* This follows from the fact that the closed set $K \cdot R^{\prime}$ is separated between $M_{i} \cdot R^{\prime}$ and $M_{j} \cdot R^{\prime}$.

$\dagger \mathrm{J}$. W. Alexander, these Transactions, loc. cit. 


$$
\Gamma^{1} \sim 0
$$

$\left(\bmod 2, E_{3}-J\right)$,

which is impossible since $\Gamma^{1}$ links $J$. Let the distance between $J$ and $\Gamma^{1}$, as ordinarily defined for point sets, be denoted by $\epsilon$. By condition (3) of the theorem, there is a number $\delta_{\epsilon}$ such that if $\Gamma^{0}$ is a closed 0 -chain of $S_{1}$ of diameter less than $\delta_{\epsilon}$, then there is a 1 -chain $\Gamma_{1}^{1}$ such that

$$
\Gamma_{1}{ }^{1} \equiv \Gamma^{0}
$$

where the diameter of $\Gamma_{1}^{1}$ is less than $\frac{1}{4} \epsilon$.

We may assume, without loss of generality, that the 1 -chain $\Gamma^{1}$ is irreducible; and that its 1-cells have a given cyclic order. We may also assume that the one-cells of $\Gamma^{1}$ are all of diameter less than $\frac{1}{4} \delta_{\epsilon}$.

The cells of $\Gamma^{1}$ may be divided into two classes, according to whether their boundaries lie wholly in $S_{1}$ or not. We may start with a given cell $c^{1}$, and consider the successive cells of $\Gamma^{1}$ in their cyclic order. If both end-cells of $c^{1}$ lie in $S_{1}$, then $c^{1}$ may be replaced by a 1 -chain $K^{1}$ which has the same boundary, but which lies entirely in $S_{1}$ and has a diameter less than $\frac{1}{4} \epsilon$. We may agree to say that the cell $c^{1}$ has been transformed into the chain-cell $K^{1}$. Proceeding to the next 1-cell of $\Gamma^{1}$, which we may denote by $d^{1}$, suppose that one of its end-cells is on $K$. Denote the cell that it has in common with $c^{1}$ by $a^{0}$, and the cell on $K$ by $b^{0}$. There is a 0 -cell, $\bar{b}^{0}$, in $S_{1}$, whose distance from $b^{0}$ is less than $\frac{1}{4} \delta_{\epsilon}$, and since the distance from $a^{0}$ to $\bar{b}^{0}$ is less than $\delta_{\epsilon}$, these two cells bound a 1 -chain $K_{1}^{1}$ in $S_{1}$, whose diameter is less than $\frac{1}{4} \epsilon$. We shall replace $b^{0}$ by $\bar{b}^{0}$, and $d^{1}$ by $K_{1}^{1}$, and say that $b^{0}$ and $d^{1}$ have been transformed, respectively, into $\bar{b}^{0}$ and $K_{1}^{1}$. Suppose the next cell on $\Gamma^{1}$ is $e^{1}$, and that its end-cell distinct from $b^{0}$, viz. $c^{0}$, is also on $K$. There is a 0 -cell $\bar{c}^{0}$ in $S_{1}$ whose distance from $c^{0}$ is less than $\frac{1}{4} \delta_{\epsilon}$. Clearly the distance from $\bar{b}^{0}$ to $\bar{c}^{0}$ is less than $\delta_{\epsilon}$, and hence these two cells bound a 1 -chain $K_{2}{ }^{1}$ in $S_{1}$ of diameter less than $\frac{1}{4} \epsilon$.We shall let $\bar{c}^{0}$ and $K_{2}^{1}$ be the transforms, respectively, of $c^{0}$ and $e^{1}$.

Proceeding through all of the cells of $\Gamma^{1}$ as just indicated, we replace each cell of $\Gamma^{1}$ by its transform, and obtain a new closed 1-chain, $\Gamma_{1}{ }^{1}$, which we may call the transform of $\Gamma^{1}$. Now $\Gamma_{1}^{1}$ lies wholly in $S_{1}$, and accordingly, by application of condition (4),

$$
\Gamma_{1}^{1} \sim 0
$$

$\left(\bmod 2, E_{3}-J\right)$.

We shall show that relation (a) is impossible.

We note that if $c^{1}$ is any 1-cell of $\Gamma^{1}$, then every point of the transform, $K^{1}$, of $c^{1}$, is at a distance less than $\frac{1}{2} \epsilon$ from either end-cell of $c^{1}$. That is, both of the chains $c^{1}$ and $K^{1}$ lie within a spherical neighborhood $T$ of radius $\epsilon$ which has its center at one end-cell of $c^{1}$ and accordingly contains no point of 
$J$. By introducing, if necessary, two new 1-chains, each bounded by an endcell of $c^{1}$ and its transform, a closed 1-chain $L^{1}$ is obtained in $T$ which contains $c^{1}$ and $K^{1}$ and such that

$$
L^{1} \sim 0 \quad\left(\bmod 2, T, E_{3}-J\right) .
$$

By adding homology (a) and all homologies of type (b), we have

$$
\Gamma_{1}{ }^{1}+\sum L^{1} \sim 0
$$

$\left(\bmod 2, E_{3}-J\right)$.

However, we have that, adding modulo 2,

$$
\Gamma_{1}^{1}+\sum L^{1}=\Gamma^{1}
$$

(it can be assumed that only one chain is introduced between each 0 -cell of $\Gamma^{1}$ and its transform). Combining relations (c) and (d) we have

$$
\Gamma^{1} \sim 0
$$

$\left(\bmod 2, E_{3}-J\right)$.

This contradicts the fact that $\Gamma^{1} \operatorname{links} J$. Hence $\Gamma^{1}$ must have points in both $S_{1}$ and $S_{2}$.

We shall now show that the supposition that $K-J$ is connected is inconsistent with the fact that $\Gamma^{1}$ links $J$, and has points in both $S_{1}$ and $S_{2}$. It will first be necessary to make a transformation of $\Gamma^{1}$.

Let $\epsilon_{1}$ be a positive number less than $\frac{1}{4} \epsilon$. Since $K$ is uniformly connected im kleinen (being a bounded, connected im kleinen continuum), there is a positive number $\epsilon_{2}$, such that if $P$ and $Q$ are points of $K$ at a distance apart less than $\epsilon_{2}$, then $P$ and $Q$ are joined by an arc of $K$ of diameter less than $\epsilon_{1}$. Let $\delta_{\epsilon}$, be a positive number such that if $P$ and $Q$ are 0 -cells of $S_{i}(i=1$ or 2$)$ at a distance apart less than $\delta_{\epsilon_{2}}$, then $P$ and $Q$ bound a 1-chain in $S_{i}$ of diameter less than $\frac{1}{4} \epsilon_{2}$. We may assume that the 1-cells of $\Gamma^{1}$ are all of diameter less than $\frac{1}{4} \delta_{\epsilon_{2}}$.

The transformation that we now effect on $\Gamma^{1}$ is very similar to the one described above, except that the new constants just defined are involved. Supposing that we start with a cell $c^{1}$ of $\Gamma^{1}$ whose end-cells are both in $S_{1}$, we replace it by a chain-cell $K^{1}$ with the same boundary and diameter less than $\frac{1}{4} \epsilon_{2}$. If the next cell $b^{1}$ has an end-cell on $K$, we transform it into a chain-cell in $S_{1}$. Of course we ultimately come to a cell $c^{1}$ one of whose endcells (the one last affected by a transformation), $a^{0}$, is in $S_{1}+K$, and the other, $b^{0}$, is in $S_{2}$. (Indeed, if $\Gamma^{1}$ had none of its bounding 0 -cells in $S_{2}$, the whole chain $\Gamma^{1}$ could be transformed into a chain in $S_{1}$, just as in the above proof, and a contradiction obtained as before. Similarly, if we encounter a case of one bounding 0 -cell of $\Gamma^{1}$ in $S_{2}\left(S_{1}\right)$ and the neighboring 0 -cells in $S_{1}\left(S_{2}\right)$, the 1-cells which they bound may be transformed into chain-cells lying in 
$S_{1}\left(S_{2}\right)$.) It is unnecessary to use condition (3) here, since any 1-chain $K^{1}$ of diameter less than $\frac{1}{4} \epsilon_{2}$ and bounded by $\tilde{a}^{0}$ and $b^{0}$ will do for the transform of $c^{1}\left(\bar{a}^{0}\right.$ being the transform of $\left.a^{0}\right)$. The next 0 -cell, $c^{0}$, is in $S_{2}$ (else we would have proceeded as indicated in the above parenthesis) and the transformation to be effected is obvious. The subsequent transformations should also be obvious, since we now proceed from $S_{2}$ in just such a manner as we proceeded from $S_{1}$, viz., by not actually "crossing" into $S_{1}$ until two successive bounding 0 -cells are encountered in $S_{1}$.

The outcome of these transformations on the cells of $\Gamma^{1}$ is to obtain a new 1-chain, which we shall still denote by $\Gamma^{1}$ and which still links $J$ (this can be shown as above), but which has the following properties: (1) its intersections with $K$ occur on certain chain-cells, $K_{1}^{1}, K_{2}^{1}, \cdots, K_{n}^{1}$ in the order named; we shall henceforth call these the crossing-cells of $\Gamma^{1}$; (2) if $K_{2}^{1}$ is any crossing-cell, the diameter of $K_{i}{ }^{1}$ is less than $\frac{1}{4} \epsilon_{2} ;(3)$ the boundary cells of $K_{i}^{1}$ lie in $S_{1}$ and $S_{2}$, respectively; (4) if $K_{i}^{1}$ and $K_{i+1}^{1}$ are bounded by the cells $a^{0}, b^{0}, c^{0}$ and $d^{0}$ (these occurring in the order named), then the cells $a^{0}$ and $d^{0}$ are both in $S_{1}\left(S_{2}\right)$; in other words, if one crossing-cell leads from $S_{1}$ to $S_{2}$, the next leads from $S_{2}$ to $S_{1}$. The last property is very important, and shows that there is an even number of crossing-cells.

We shall now proceed to replace $\Gamma^{1}$ by a 1 -chain which still links $J$ but has only two crossing-cells.

Starting with $K_{1}{ }^{1}$, suppose that the bounding 0 -cells of $K_{1}{ }^{1}$ and $K_{2}{ }^{1}$ occur in the order $a^{0}, b^{0}, c^{0}, d^{0}$, where $a^{0}$ is in $S_{1}$. In $S_{1}$, let $A_{1}^{1}$ be a new 1-chain bounded by $a^{0}$ and $d^{0}$, and in $S_{2}$ let $A_{2}{ }^{1}$ be the 1-chain of $\Gamma^{1}$ which lies in $S_{2}$ and is bounded by $b^{0}$ and $c^{0}$. Denote the portion of $\Gamma^{1}$ from $d^{0}$ to $a^{0}$ by $B^{1}$. Now we cannot have both of the following relations:

$$
\begin{aligned}
& A_{1}^{1}+K_{1}^{1}+A_{2}^{1}+K_{2}^{1} \sim 0, \\
& A_{1}^{1}+B^{1} \sim 0
\end{aligned}
$$

since the sum of these homologies would imply the homology

$$
\Gamma^{1} \sim 0 \quad\left(\bmod 2, E_{3}-J\right) .
$$

If (e) fails to hold, we have secured the type of 1-chain linking $J$ that we set out to obtain, viz., a chain with only two crossing-cells. If (e) holds, we can proceed with the 1-chain

$$
\Gamma^{1}+K_{1}{ }^{1}+A_{2}{ }^{1}+K_{2}{ }^{1}+A_{1}{ }^{1}=A_{1}{ }^{1}+B^{1}
$$

which has two less crossing-cells than $\Gamma^{1}$, viz., $K_{3}^{1}, \cdots, K_{n}{ }^{1}$, by next considering the crossing-cells $K_{3}^{1}$ and $K_{4}^{1}$.

In any case, we eventually arrive at a closed 1-chain which links $J$, and 
has only two of the original crossing-cells of $\Gamma^{1}$. We shall continue to call this chain $\Gamma^{1}$, and we shall suppose its crossing-cells are $K_{1}^{1}$ and $K_{2}^{1}$, their boundary cells being denoted, as before, by $a^{0}, b^{0}, c^{0}, d^{0}$. Denote the portion of $\Gamma^{1}$ lying in $S_{1}$ and bounded by $a^{0}+d^{0}$ by $L_{1}^{1}$ and the portion in $S_{2}$ bounded by $b^{0}+c^{0}$ by $L_{2}^{1}$.

Because of the way in which the above transformation was carried out, at least one of the boundary cells of $K_{i}{ }^{1}(i=1,2)$ is identical with a cell of the original chain $\Gamma^{1}$, so that a spherical neighborhood $T_{i}$ of diameter $\epsilon_{2}$ about $K_{i}^{1}$ certainly encloses no points of $J$. We may assume that $K_{1}^{1}$ and $K_{2}{ }^{1}$ are homeomorphic with simple arcs, and on $K_{i}{ }^{1}(i=1,2)$ let $x_{i}$ and $y_{i}$ be points of $K$ such that the portions $a^{0} x_{1}\left(d^{0} x_{2}\right)$ and $b^{0} y_{1}\left(c^{0} y_{2}\right)$ of $K_{1}{ }^{1}\left(K_{2}^{1}\right)$ contain no points of $K$ other than $x_{1}$ and $y_{1}\left(x_{2}\right.$ and $\left.y_{2}\right)$.

Since $x_{1}$ and $x_{2}$ lie in $K-J$, and $K-J$ is connected, there is an $\operatorname{arc} x_{1} x_{2}$ in $K-J .^{*}$ Denote the distance between $x_{1} x_{2}$ and $J$ by $\epsilon_{3}$, let $\epsilon_{4}$ denote a positive number less than both $\epsilon_{1}$ and $\epsilon_{3}$, and let $\delta_{\epsilon_{4}}$ be a positive number such that if two 0-cells of $S_{i}$ are at a distance apart less than $\delta_{\epsilon_{4}}$, they bound a 1-chain in $S_{i}$ of diameter less than $\frac{1}{4} \epsilon_{4}$ (condition (3)).

Let $F_{1}, F_{2}, \cdots, F_{m}$ be points on the arc $x_{1} x_{2}$, where $F_{1}=x_{1}$ and $F_{m}=x_{2}$, occurring in the order named, and so selected that

$$
\delta\left(F_{i} F_{i+1}\right)<\frac{1}{4} \delta_{e_{4}} \quad(i=1,2, \cdots, m-1),
$$

where $F_{i} F_{i+1}$ denotes the portion of $x_{1} x_{2}$ between $F_{i}$ and $F_{i+1}$. Let $P_{1}, P_{2}, \cdots$, $P_{m}$ be a set of 0-cells in $S_{1}$ such that $P_{1}$ is on the portion $a^{0} x_{1}$ of $K_{1}^{1}$ and $P_{m}$ on the portion $d^{0} x_{2}$ of $K_{2}^{1}$, and the following relations hold:

$$
\begin{aligned}
\rho\left(P_{i}, F_{i}\right) & <\frac{1}{4} \epsilon_{4}, \\
\rho\left(P_{i}, P_{i+1}\right) & <\delta_{\epsilon 4} .
\end{aligned}
$$

According to the definition of $\delta_{\epsilon_{4}}$, there exists for each $i$ an open 1-chain $H_{i}^{1}$ in $S_{1}$ bounded by $P_{i}$ and $P_{i+1}$, and such that $\delta\left(H_{i}{ }^{1}\right)<\frac{1}{4} \epsilon_{4}$. We define $C_{1}^{1}=\sum_{i=1}^{m-1} H_{i}^{1}$, and we also note that every point of $H_{i}{ }^{1}$ lies within a distance $\frac{1}{2} \epsilon_{4}$ of $F_{i}$.

Let $T_{i}^{1}(i=1,2)$ be a spherical neighborhood concentric with $T_{i}$ and of diameter $2 \epsilon_{1}$. Clearly $T_{i}^{1}$ contains no point of $J$. According to the definition of $\epsilon_{2}$, and because of the fact that the distance from $x_{i}$ to $y_{i}$ is less than $\epsilon_{2}$ (the diameter of $K_{i}{ }^{1}$ being less than $\epsilon_{2}$ ), there is an $\operatorname{arc} t_{i}$ in $K$ which lies wholly in $T_{i}$ and has end points $x_{i}$ and $y_{i}$. We can approximate the arc $t_{1}$ by a $\frac{1}{4} \delta_{e_{4}}$-chain $\dagger$ of 0 -cells in $S_{2}$, with first point, $z_{1}$, on the portion $y_{1} b^{0}$ of $K_{1}^{1}$, and

* R. L. Moore, Mathematische Zeitschrift, loc. cit.

$\dagger$ The word "chain" is here used in the ordinary point-set theoretic sense; i.e., if $\epsilon$ is a positive number, and $P$ and $Q$ are points, an $\epsilon$-chain from $P$ to $Q$ is a set of points $P_{1}, P_{2}, \cdots, P_{n}$, where $P_{1}=P$ and $P_{n}=Q$, and $\rho\left(P_{i}, P_{i+1}\right)<\epsilon(i=1,2, \cdots, n-1)$. 
last point, $z_{h}$, and such that not only is every point of the chain at a distance less than $\frac{1}{4} \epsilon_{4}$ from some point of $t_{1}$, but in particular $\rho\left(z_{h}, F_{1}\right)<\frac{1}{4} \epsilon_{4}$. Then, in a manner similar to that used in obtaining $C_{1}{ }^{1}$, we obtain a 1 -chain $B_{1}{ }^{1}$ bounded by $z_{1}+z_{h}$, lying wholly in $S_{2}$, and such that every point of $B_{1}{ }^{1}$ is within a distance $\frac{1}{2} \epsilon_{4}$ from some point of $t_{1}$. It is clear that a spherical neighborhood $T_{1}{ }^{2}$ concentric with $T_{1}$ and of diameter $3 \epsilon_{1}$, will contain $B_{1}{ }^{1}$, and enclose no points of $J$.

We can obtain similarly a 1-chain $B_{2}^{1}$ with reference to $t_{2}$, so that the boundary of $B_{2}{ }^{1}$ is a point $w_{1}$ on the portion $y_{2} c^{0}$ of $K_{2}{ }^{1}$ and a point $w_{k}$ in $S_{2}$ whose distance from $F_{m}$ is $<\frac{1}{4} \epsilon_{4}$. Let $T_{2}^{2}$ be a spherical neighborhood analogous to $T_{1}^{2}$.

Let $Q_{1}, Q_{2}, \cdots, Q_{m}$ be points in $S_{2}$ chosen with reference to the points $\left\{F_{i}\right\}$ just as the points $\left\{P_{i}\right\}$ were chosen in $S_{1}$, and so that $Q_{1}=z_{h}$ and $Q_{m}=w_{k}$. On these points can be built up a 1-chain $B_{3}{ }^{1}$ of $S_{2}$, bounded by $Q_{1}$ and $Q_{m}$, and approximating the arc $x_{1} x_{2}$ in a manner similar to that in which $C_{1}^{1}$ approximates $x_{1} x_{2}$.

We now define the following 1-chains:

$$
\begin{aligned}
& C_{2}{ }^{1}=\sum_{i=1}^{3} B_{i}{ }^{1}, \\
& \Gamma_{1}^{1}=L_{1}^{1}+a^{0} P_{1}+C_{1}^{1}+P_{m} d^{0}, \\
& \Gamma_{2}^{1}=C_{1}^{1}+P_{1} z_{1}+C_{2}{ }^{1}+w_{1} P_{m}, \\
& \Gamma_{3}^{1}=C_{2}^{1}+z_{1} b^{0}+L_{2}{ }^{1}+c^{0} w_{1},
\end{aligned}
$$

where $a^{0} P_{1}, P_{m} d^{0}$, etc., are portions of $K_{1}^{1}$ and $K_{2}^{1}$. We note that

$$
\Gamma^{1}=\sum_{i=1}^{8} \Gamma_{i}^{1}
$$

The following homologies follow from condition (4) of the theorem:

$$
\begin{aligned}
& \Gamma_{1}^{1} \sim 0, \\
& \Gamma_{3}^{1} \sim 0
\end{aligned}
$$$$
\left(\bmod 2, E_{3}-J\right) .
$$

Consequently, since $\Gamma^{1}$ links $J$, it follows from relations (g), (h) and (i) that $\Gamma_{2}{ }^{1}$ links $J$.

If we join $P_{1}$ and $z_{h}$ by a 1 -chain $B_{4}{ }^{1}$ every point of which is at a distance less than $\frac{1}{4} \epsilon_{4}$ from $x_{1}\left(=F_{1}\right)$ and $P_{m}$ and $w_{k}$ similarly by a 1 -chain $B_{5}{ }^{1}$, it is easy to see that since the following relations hold:

$$
\begin{aligned}
B_{1}{ }^{1}+B_{4}{ }^{1}+P_{1} z_{1} \sim 0 & \left(T_{2}^{1}, E_{3}-J\right), \\
B_{2}{ }^{1}+B_{5}{ }^{1}+P_{m} w_{1} \sim 0 & \left(T_{2}{ }^{2}, E_{3}-J\right),
\end{aligned}
$$


the closed chain

$$
\Gamma=C_{1}^{1}+\sum_{i=3}^{5} B_{i}^{1}
$$

links $J$.

However, this is impossible for the following reasons. The points $P_{i}$ and $Q_{i}(i=2,3, \cdots, m-1)$ bound a 1 -chain $N_{i}{ }^{1}$, every point of which is at a distance less than $\frac{1}{4} \epsilon_{4}$ from $F_{i}$. For convenience of notation, let $N_{1}^{1}=B_{4}^{1}$ and $N_{m}^{1}=B_{5}{ }^{1}$. Let $U_{i}(i=1,2, \cdots, m)$ be a spherical neighborhood of $F_{i}$ of radius $\epsilon_{4}$. Then every point of the closed chain

$$
E_{i}^{1}=H_{i}^{1}+N_{i}{ }^{1}+\bar{H}_{i}^{1}+N_{i+1}^{1} \text {, }
$$

where $\bar{H}_{i}{ }^{1}$ is the portion of $B_{3}{ }^{1}$ from $Q_{i}$ to $Q_{i+1}$, lies in $U_{i}$. Hence

$$
E_{i}^{1} \sim 0
$$

$\left(\bmod 2, E_{3}-J\right)$.

But

$$
\Gamma_{-}=\sum_{i=1}^{m} E_{i}^{1}
$$

and by adding all homologies $(\mathrm{j})$, we get

$$
\Gamma \sim 0
$$

$\left(\bmod 2, E_{3}-J\right)$.

Thus, on the assumption that $K-J$ is connected, we are able to find a closed chain $\Gamma$, homologous to the chain $\Gamma^{1}$ which links $J$, but which does not itself link $J$. As this is impossible, $K-I$ cannot be connected.

VI. If $J$ is a simple closed curve of $K$, then $K-J$ contains at most two components. For suppose $K-J$ contains at least 3 components, $M_{1}, M_{2}$, and $M_{3}$. Since $K$ is a continuous curve, each component of $K-J$ is also a quasicomponent of $K-J,{ }^{*}$ and it is therefore easy to see, with the result of section IV, that each component of $K-J$ has all of $J$ as its boundary.

There exists on $J$ a set of points occurring in the following order, $P_{1} A_{1} Q_{1} B_{1} P_{2} B_{2} Q_{2} A_{2} P_{1}$, and such that there is an $A_{i} x_{i} B_{i}(i=1,2)$ lying, except for $A_{i}$ and $B_{i}$, wholly in $M_{i}$. Define a simple closed curve $J_{1}$ as follows:

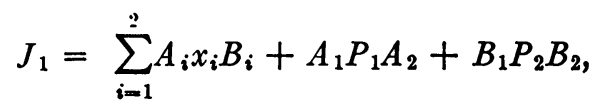

where $A_{1} P_{1} A_{2}$ and $B_{1} P_{2} B_{2}$ are arcs of $J$. Then the set $K-J_{1}$ is connected, in contradiction to the result of section $V$. For if we denote the set

* Cf. R. L. Wilder, A characterization of continuous curves by a property of their open subsets, Fundamenta Mathematicae, vol. 11 (1928), pp. 127-131. 
$K-\left(M_{1}+M_{2}+J\right)$ by $R$, it is clear that the set $R_{1}=R+\sum_{i=1}^{2}<A_{i} Q_{i} B_{i}>$ is connected. Also, the set $M_{1}-\left\langle A_{1} x_{1} B_{1}\right\rangle$ contains only components that have boundary points on either $\left\langle A_{1} Q_{1} B_{1}\right\rangle$ or $\left\langle A_{2} Q_{2} B_{2}\right\rangle$. For suppose $M_{1}$ $\left.-<A_{1} x_{1} B_{1}\right\rangle$ contains a component $C$ with boundary points only on the set $t=A_{2} P_{1} A_{1} x_{1} B_{1} P_{2} B_{2}$. Then the set

$$
K-t=C+\left[\left(M_{1}-<A_{1} x_{1} B_{1}>-C\right)+M_{2}+R+\sum_{i=1}^{2}<A_{i} Q_{i} B_{i}>\right]
$$

is not connected, contrary to the result of IV. In a like manner it is shown that all components of $M_{2}-A_{2} x_{2} B_{2}$ have limit points on $\left.\sum_{i=1}^{2}<A_{i} Q_{i} B_{i}\right\rangle$, and it is clear that the set

$$
K-J_{1}=R_{1}+\sum_{i=1}^{2}\left(M_{i}-A_{i} x_{i} B_{i}\right)
$$

is connected.

VII. Denoting the two components of $K-J$ by $M_{1}$ and $M_{2}$, respectively, the sets $M_{1}$ and $M_{2}$ are uniformly connected im kleinen. For suppose $M_{1}$ is not uniformly connected im kleinen. Then there is a point $P$ on $J$, a spherical neighborhood $R$ of $P$, and a sequence of pairs of points of $M_{1},\left\{P_{n}, Q_{n}\right\}$, such that $\lim _{n \rightarrow \infty} P_{n}=P$ and $\lim _{n \rightarrow \infty} Q_{n}=P$, and for no $n$ are $P_{n}$ and $Q_{n}$ joined in $R$ by any connected subset of $M_{1}$. Denote the set $M_{i} \cdot R$ by $\bar{M}_{i}(i=1,2)$, and the frontier of $R$ by $F$.

Denote by $C$ the component of $K \cdot R$ determined by $P$. Let $R_{1}$ be a spherical neighborhood of $P$ of smaller radius than $R$, which encloses no points of $J$ that are not on the arc, $t$, component of $J \cdot R$ determined by $P$. We shall denote $J \cdot R$ by $t_{1}$.

Let $R_{2}$ be another spherical neighborhood of $P$ such that any point of $K$ within $R_{2}$ is joined to $P$ by an $\operatorname{arc}$ of $K$ that lies in $R_{1}$ and such that $R_{2}$ encloses no points of $K-C$. Let $P_{k}, Q_{k}$ be a pair of points of the sequence $\left\{P_{n}, Q_{n}\right\}$ lying in $R_{2}$, and let the component of $\bar{M}_{1}$ determined by $P_{k}$ be denoted by $H_{1}$; let $\bar{M}_{1}-H_{1}=H_{2}$. Then $H_{1}$ and $H_{2}$ are mutually separated.

In $R_{2}$, let $x_{1}$ and $x_{2}$ be points of $S_{1}$ and $S_{2}$, respectively, that are 0 -cells of the subdivision of $E_{3}$.

(a) The set $C$ separates $R$ between $x_{1}$ and $x_{2}$. For $x_{1}$ and $x_{2}$ bound a chain $\Gamma_{1}^{1}$ in $E_{3}-[F+(K-C)]$, and if they bound a chain $\Gamma_{2}^{1}$ in $E_{3}-(F+C)$, then, since the closed chain $\Gamma_{1}^{1}+\Gamma_{2}^{1}$ is homologous to zero in $R$, it cannot link the product $[F+(K-C)] \cdot[F+C]$, which is a subset of $F$, and accordingly $x_{1}+x_{2}$ is homologous to zero in $E_{3}-K$ (Alexander's Corollary $W_{i}$, loc. cit.). This is a contradiction of condition (1) of the theorem.

(b) Under the assumption that $M_{1}$ is not uniformly connected im kleinen 
in the neighborhood of $P, C$ does not separate $x_{1}$ and $x_{2}$ in $R$. Let $y_{1}$ and $y_{2}$ be the first points of $K$ on a straight line from $x_{1}$ to $x_{2}$, in the orders $x_{1}$ to $x_{2}$ and $x_{2}$ to $x_{1}$, respectively. There exist $\operatorname{arcs} y_{1} P_{k}, y_{1} Q_{k}, y_{2} P_{k}$ and $y_{2} Q_{k}$ of $K$ in $R_{1}$. By the use of the usual approximation based on condition (3) of the theorem, we obtain a closed 1-chain, $\Gamma^{1}$, consisting of two open chains $\Gamma_{1}^{1}$ and $\Gamma_{2}^{1}$ bounded by $x_{1}$ and $x_{2}$, such that $\Gamma^{1}$ lies in $R_{1}$, and cuts $K$ only in certain arbitrarily small neighborhoods of $P_{k}$ and $P_{j}$, respectively. The following congruences hold:

$$
\begin{array}{lr}
\Gamma_{1}^{1} \equiv x_{1}+x_{2} & {\left[\bmod 2, E_{3}-\left(F+H_{1}^{\prime}\right)\right]} \\
\Gamma_{2}{ }^{1} \equiv x_{1}+x_{2} & {\left[\bmod 2, E_{3}-\left(F+H_{2}^{\prime}+\bar{M}_{2}^{\prime}\right)\right]}
\end{array}
$$

(where $H_{1}^{\prime}$ denotes $H_{1}$ together with all its limit points, etc.). We may assume that the product of $H_{1}^{\prime}$ and $H_{2}^{\prime}$ is a subset of $t_{1}^{\prime}$, so that if we can show that $\Gamma^{1}$ bounds in $E_{3}-\left(F+H_{1}^{\prime}\right) \cdot\left(F+H_{2}^{\prime}+\bar{M}_{2}^{\prime}\right)$ or, since the latter set contains $E_{3}-\left(F+t_{1}\right)$, if we can show that $\Gamma^{1}$ bounds in $E_{3}-\left(F+t_{1}\right)$, we shall have that $x_{1}$ and $x_{2}$ are not separated in $R$ by $C$ (Alexander's Corollary $W_{i}$, loc. cit.).

Since $M_{1}$ is connected, it can be shown, by the methods employed in section $\mathrm{V}$, that $\Gamma^{1}$ does not link $J$. Consequently, there exists a chain $\Gamma_{1}{ }^{2}$ such that

$$
\Gamma_{1}^{2} \equiv \Gamma^{1} \quad\left(\bmod 2, E_{3}-J, E_{3}-t^{\prime}\right) .
$$

But since $\Gamma^{1}$ lies in $R_{1}$, and the latter contains no points of $F+J-t$, we have

$$
\Gamma_{2}^{2} \equiv \Gamma^{1} \quad\left[\bmod 2, E_{3}-(F+J-t)\right] .
$$

Now since the product of $F+J-t$ and $t^{\prime}$ is just two points, and neither $\Gamma_{1}{ }^{2}$ nor $\Gamma_{2}{ }^{2}$ meets the arc $J-t$ joining these points, we have that

$$
\Gamma_{1}^{2}+\Gamma_{2}^{2} \sim 0 \quad\left[\bmod 2, E_{3}-t^{\prime} \cdot(F+J-t)\right] .
$$

Hence, by Alexander's Corollary $W_{i}$ (loc. cit.),

$$
\Gamma^{1} \sim 0 \quad\left[\bmod 2, E_{3}-\left(F+t_{1}\right)\right] .
$$

As this is the relation we wished to prove, in order to show that the 0 -chain $x_{1}+x_{2}$ bounds in $R-C$, we have shown that $x_{1}$ and $x_{2}$ are not separated by $C$ in $R$.

As (a) and (b) are in contradiction, the assumption that $M_{1}$ is not uniformly connected im kleinen cannot hold. A like statement of course holds for $M_{2}$, and the theorem is proved.

The following theorem follows immediately from Theorem 3, from which it is only slightly different: 
Theorem 4 (Converse of the Jordan-Brouwer Separation Theorem in $\left.E_{3}\right)$. Let $K$ be a closed and bounded set in $E_{3}$, such that (1) the Betti numbers $(\bmod 2) R^{0}\left(E_{3}-K\right)$ and $R^{1}\left(E_{3}-K\right)$ are respectively equal to 2 and $1,(2)$ if $D$ is a component of $E_{3}-K$, the 0 -chains in $D$ are uniformly homologous to zero in $D$, and every point of $K$ is a limit point of $D$. Then $K$ is a simple closed surface.

Since there are two components, $S_{1}$ and $S_{2}$, in $E_{3}-K$, condition (1) of Theorem 3 is satisfied. Since every point of $K$ is a limit point of each of these components, condition (2) of Theorem 3 is satisfied; for, it is to be noted, condition (2) of Theorem 3 allows $P^{\prime}$ to lie in that complementary domain which contains $Q$. Conditions (3) and (4) of Theorem 3 are restated in Theorem 4. Thus Theorem 4 follows at once from Theorem 3 .

4. Appendix. The independence of the conditions of Theorem 3 is established as follows:

Condition (1): In $E_{3}$, using rectangular coördinates, let $K$ be the set of points $(x, y, 0)$ such that $x^{2}+y^{2} \leqq 1$. Let $S_{1}$ be the set of all points for which $z>0$; let $S_{2}=E_{3}-\left(K+S_{1}\right)$.

Condition (2): Using spherical coördinates, let $K$ be the set of all points $(\rho, \phi, \theta)$ for which $1 \leqq \rho \leqq 2$, let $S_{1}$ be the set of points for which $\rho<1$, and $S_{2}$ the set for which $\rho>2$.

Condition (3): Using spherical coördinates, let $K^{\prime}$ denote the set of points $\rho=1$. On $K^{\prime}$, let $t$ denote the arc consisting of points $(1,0, \theta)$ such that $0 \leqq \theta \leqq \pi$. On $t$, if $P$ denotes any point $(1,0, \theta)$, let $P^{\prime}$ denote the point $(1,0, \pi-\theta)$. The set $K$ is obtained by continuously deforming $K^{\prime}$ so that each point $P$ coincides with $P^{\prime}$, but points not on $t$ remain distinct as before. The complement of $K$ is two domains, neither of which satisfies condition (3) of Theorem 3.

Condition (4): The torus.

The equivalence, in the case of open sets in $E_{n}$, of the conditions uniformly homologous to zero and uniformly connected im kleinen, is established in the following theorem:

THEOREM 5. In order that an open set $G$ in $E_{n}$ should be uniformly connected im kleinen, it is necessary and sufficient that the 0-chains in $G$ should be uniformly homologous to zero in $G$.

The condition is necessary. Let $\epsilon$ be any positive number. Since $G$ is uniformly connected im kleinen, there exists a positive number $\delta$ such that if $P$ and $Q$ are 0 -cells of $G$ whose distance apart is less than $\delta$, there is an arc $P Q$ of $G$ whose diameter is less than $\frac{1}{4} \epsilon$. 
Let $E_{n}-G=F$, and denote the distance between $F$ and the arc $P Q$ by $\eta_{1}$. Let $\eta$ be a positive number smaller than either of the numbers $\eta_{1}, \frac{1}{2} \epsilon$. Let $P_{1}(=P), P_{2}, \cdots, P_{n}(=Q)$ be points of $P Q$ such that $\rho\left(P_{i}, P_{i+1}\right)<\frac{1}{4} \eta(i=1$, $2, \cdots, n-1)$. The subdivision of $E_{n}$ may be extended so that there exist 0-cells $F_{1}\left(=P_{1}\right), F_{2}, \cdots, F_{n}\left(=P_{n}\right)$ such that $\rho\left(F_{i}, F_{i+1}\right)<\frac{1}{4} \eta$ and $\rho\left(F_{i}, P_{i}\right)$ $<\frac{1}{4} \eta$. Let $T_{i}$ be a spherical neighborhood of $P_{i}$ of radius $\frac{1}{2} \eta$. Then clearly $F_{i+1}$ lies in $T_{i}$, and consequently $F_{i}+F_{i+1}$ bounds a 1-chain $H_{i}$ in $T_{i}$. Since no point of $F$ lies in $T_{i}, H_{i} \cdot F=0$. Consequently the chain $K^{1}=\sum_{=1}^{n-1} H_{i}$ lies in $G$.

The diameter of $K^{1}$ is less than $\epsilon$. For let $x_{1}$ and $x_{2}$ be points of $K^{1}$. Since $x_{1}$ lies in some sphere $T_{i}$, and hence $\rho\left(x_{1}, P_{i}\right)<\frac{1}{2} \eta<\frac{1}{4} \epsilon$, and since there exists, similarly, a $P_{j}$ such that $\rho\left(x_{2}, P_{j}\right)<\frac{1}{4} \epsilon$, it follows at once from the fact that the diameter of $P Q$ is $<\frac{1}{4} \epsilon$, that $\rho\left(x_{1}, x_{2}\right)<\epsilon$.

The condition is sufficient. If $\epsilon$ is a positive number, there is a positive number $\delta$ such that if $x_{1}{ }^{0}$ and $x_{2}{ }^{0}$ are 0 -cells of $G$ such that $\rho\left(x_{1}^{0}, x_{2}{ }^{0}\right)<\delta$, then $x_{1}{ }^{0}+x_{2}{ }^{0}$ bounds a chain $K^{1}$ of $G$ of diameter $<\frac{1}{4} \epsilon$. Let $P_{1}$ and $P_{2}$ be any two points of $G$ such that $\rho\left(P_{1}, P_{2}\right)<\frac{1}{4} \delta$. The subdivision of $E_{n}$ may be extended so that there exist 0 -cells $y_{1}{ }^{0}$ and $y_{2}{ }^{0}$ such that $\rho\left(x_{i}{ }^{0}, P_{i}\right)<\frac{1}{4} \delta(i=1,2)$, and such that there are $\operatorname{arcs} y_{i} P_{i}$ of diameter $<\frac{1}{4} \epsilon$ in $G$. Since $\rho\left(y_{1}^{0}, y_{2}{ }^{0}\right)<\delta$, $y_{1}{ }^{0}+y_{2}{ }^{0}$ bounds a chain $K^{1}$ of $G$ of diameter $<\frac{1}{4} \epsilon$. The set $K^{1}+\sum_{i=1}^{2} y_{i} P_{i}$ contains an $\operatorname{arc}$ from $P_{1}$ to $P_{2}$ of diameter $<\epsilon$.

As a result of Therem 5 we can restate Theorem 4 as follows:

Theorem 4'(Converse of the Jordan-Brouwer Separation Theorem in $\left.E_{3}\right)$. In $E_{3}$, the common boundary, $K$, of two uniformly connected im kleinen domains, one of which is bounded, is a simple closed surface, provided that the Betti number $(\bmod 2) R^{1}\left(E_{3}-K\right)=1$.

The problem in $n$ dimensions, $n>3$. Regarding a converse of the JordanBrouwer Separation Theorem in $E_{n}$, where $n>3$, the author will not hazard any guesses here, but merely indicate the likelihood that by an extension of the conditions of Theorem 4, such a converse may be obtained. Thus, condition (1) of Theorem 4 may be replaced, as a result of the Alexander duality theorem, by the condition that the Bet ti numbers $R^{0}\left(E_{3}-K\right)$ and $R^{i}\left(E_{3}-K\right)$ $(i=1,2, \cdots, n-2)$ shall be respectively equal to 2 and 1 , and condition (2) may be replaced by the statement that the closed $i$-chains $(i=0,1, \cdots$, $n-2)$ of $D$ are uniformly homologous to zero in $D$, retaining, of course, the statement that every point of $K$ is a limit point of $D$. The validity of such a condition (2) is established by the following theorems, with which we close the present paper. (The extension of the definition of "uniformly homologous to zero" to $i$-chains where $i>0$ should be obvious.) 
Theorem 6. Let $M^{n-1}$ be an $(n-1)$-sphere immersed in $E_{n}$, and let $D$ be one of the domains of $E_{n}-M^{n-1}$. Then the closed $i$-chains $(n>i>0)$ of $D$ are uniformly homologous to zero in $D$.

Suppose the conclusion of the theorem untrue. Then there is a point $P$ of $M^{n-1}$ and a contracting sequence of $i$-chains of $D, K_{1}{ }^{i}, K_{2}{ }^{i}, K_{3}{ }^{i}, \cdots$, such that $P$ is the sequential limit point of any set of points $P_{1}, P_{2}, P_{3}, \cdots$, where $P_{n}$, for every $n$, is a point of $K_{n}^{i}$, and such that there is a positive number $\epsilon$ for which $K_{n}{ }^{i}$, for every $n$, fails to bound any $(i+1)$-chain of $D$ of diameter less than $\epsilon$.

Let $S(P, \epsilon / 2)$ be a spherical neighborhood of $P$ of diameter $\epsilon / 2$. Then there is an $(n-1)$-cell, $C^{n-1}$, of $M^{n-1}$, which contains $P$ as an interior point and lies wholly in $S(P, \epsilon / 2)$. Let $R$ denote a spherical neighborhood of $P$ which encloses only points of $M^{n-1}$ that lie interior to $C^{n-1}$. Denote the boundaries of $S(P, \epsilon / 2)$ and $C^{n-1}$ by $F(P, \epsilon / 2)$ and $C^{n-2}$, respectively. The set $C^{n-2}$ is of course an $(n-2)$-sphere immersed in $E_{n}$.

Let us first consider the case where $i<n-1$.

There is a number $j$, such that $K_{j}{ }^{i}$ lies wholly in $R$. Then, denoting by $B^{n-1}$ the set of all points of $M^{n-1}$ that are not interior points of $C^{n-1}$, we have the following congruences:

$$
\begin{aligned}
& L_{1}{ }^{i+1} \equiv K_{j}{ }^{i} \quad\left(\bmod 2, E_{n}-C^{n-1}\right), \\
& L_{2}^{i+1} \equiv K_{j}^{i} \quad\left\{\bmod 2, R, E_{n}-\left[B^{n-1}+F(P, \epsilon / 2)\right]\right\} .
\end{aligned}
$$

Since $i>0, i+1$ is greater than 1 , and since by Alexander's Theorem $X^{i}$ an $(n-2)$-sphere immersed in $E_{n}$ can be linked only by a 1-chain, the following homology holds:

$$
L_{1}{ }^{i+1}+{L_{2}}^{i+1} \sim 0 \quad\left(\bmod 2, E_{n}-C^{n-2}\right) .
$$

As $C^{n-2}$ is the common part of $C^{n-1}$ and $B^{n-1}+F(P, \epsilon / 2)$, it follows from Alexander's Corollary $W^{i}$ that

$$
K_{j}^{i} \sim 0 \quad[\bmod 2, D \cdot S(P, \epsilon / 2)] .
$$

As this contradicts our assumption, the theorem is proved for $i<n-1$.

If $i=n-1$, the proof is trivial. Each of the chains $K_{j}{ }^{i}$ may be assumed irreducible (i.e., $R^{i}\left(K_{j}{ }^{i}\right)=2$ ), and thus, by Alexander's Theorem $Y$, separates $E_{n}$ into just two domains, one of which, $D_{1}$, must lie in $R$. The domain $D_{1}$ is an open $n$-chain bounded by $K_{j}{ }^{i}$ and containing no point of $M^{n-1}$.

A similar proof, together with the result of Theorem 1a, yields the following general result:

Theorem 7. Let $M^{n-1}$ be any $(n-1)$-manifold immersed in $E_{n}, D$ a domain complementary to $M^{n-1}$, and $P$ a point of $M^{n-1}$ which can be considered as 
interior to an $(n-1)$-cell of definition of $M^{n-1}$. Then if $U$ is a neighborhood of $P$ bounded by an $(n-1)$-sphere and enclosing only points of $M^{n-1}$ that are interior to $C^{n-1}$, there exists, in $U$, a neighborhood $V$ of $P$ such that any $i$-chain $(i=0,1, \cdots, n-1)$ that lies in $D \cdot V$ bounds an $(i+1)$-chain of $D \cdot U$. If $M^{n-1}$ is of the type such that every one of its points can be considered as lying interior to an $(n-1)$-cell of definition of the manifold, then the $i$-chains $(i=0$, $1, \cdots, n-1)$ of $D$ are uniformly homologous to zero in $D$.

UNIVERSITY OF MICHGAN, ANn ARbor, Mich. 